\title{
Circularly polarized images with contrast reversal using pseudo-chiral metasurfaces
}

\author{
Thomas Sang Hyuk Yoo, ${ }^{\dagger}$ Johann Berthelot, ${ }^{\ddagger}$ Geraldine Guida, $₫$ Dominique \\ Demaille,$^{\S}$ Enric Garcia-Caurel, ${ }^{\dagger}$ Nicolas Bonod ${ }^{\ddagger}$ and Bruno Gallas ${ }^{*}$, \\ $\dagger$ †PICM, CNRS, Ecole Polytechnique, Université Paris-Saclay, 91128 Palaiseau, France \\ $\ddagger$ Aix Marseille Univ, CNRS, Centrale Marseille, Institut Fresnel, Marseille, France \\ ๆLEME, EA 4416, Université Paris Nanterre \\ $\S$ Sorbonne Université, Faculté des Sciences et Ingéniérie, CNRS-UMR 7588, Institut des \\ NanoSciences de Paris, F-75005, Paris, France \\ E-mail: bruno.gallas@insp.jussieu.fr
}

\begin{abstract}
Anticounterfeiting technologies rely on visual effects that are specific to particular structures and concealed to the unwary observer. Plasmonic nanostructures are one solution to these two requirements and beside colored effects, the possibility to design covert images in a particular polarization state of light is particularly appealing. The generation of circular polarization provides a new degree of freedom and the use of pseudo-chiral plasmonic resonators hosting magneto-electric resonances allows tailoring opposite handedness of circular polarization within the same nanostructure. In this work, we have defined a palette of luminance observable only in circular dichroism by engineering the shapes and positions of the resonators. The spatial control of the polarization state of light is highlighted by realizing a micrometer scale image revealed only in circular polarization with an additional contrast reversal associated with circular dichroism reversal.
\end{abstract}


Keywords : Magneto-electric coupling, plasmonics, circular polarization, images 
Metallic nanoscatterers can resonantly interact with the incoming light thanks to the excitation of localized surface plasmon resonances. ${ }^{1}$ As a consequence of the reduced spectral range over which the localized surface plasmon interacts with light, vivid colored effects can be produced referred to as plasmonic colors. Plasmonic colors in combination with grating effects have been proposed as alternatives to pigments and are particularly promising for in anti-counterfeiting applications. Dielectric nanostructures have been proposed recently to produce colors, ${ }^{2-4}$ however the most extensive work concerning polarization effect have used metallic nanostructures. The spectral response of the metallic nanoscatterers depends on the metal used, on the geometry and on the environment. All these parameters have been varied and offer degrees of freedom to engineer color palettes with subwavelength resolution and extended gamut. ${ }^{5,6}$ In addition to subwavelength resolution, polarization conversion in $1 \mathrm{D}$ gratings has also been used to design covert images or to modify their color rendering. ${ }^{7,8}$ Asymmetric resonators have also been used to intricate different images in the same pattern and at different wavelengths. ${ }^{9-11}$ Besides sub-wavelength resolution and extended gamut, one keypoint in anti-counterfeiting systems is to produce effects that are specific to one structure and that would not be reproduced by lowcost process like ink-jet printing. In that respect, it would be greatly beneficial to design covert images in circular polarization instead of linear ones. It is known that chiral plasmonic resonators or chiral stacks of resonators can exhibit linear optical activity ${ }^{12-15}$ as well as non-linear optical activity. ${ }^{16,17}$ Pseudochiral resonators are non-chiral resonators exhibiting an optical activity which originates in magneto-electric coupling. ${ }^{18-21}$ Their constant orientation yields an additive effect resulting in a net optical activity. ${ }^{22-26}$ A characteristic unique to pseudo-chiral resonators is that the sign of the optical activity depends on the direction of propagation of the incoming light. Among the different types of resonators that exhibit this property, U-shaped resonators are the most commonly used since they feature strong magneto-electric coupling and are easy to fabricate. ${ }^{18,21,28,31}$ We have shown recently that the extinction cross-section associated with magneto-electric modes in the visible is only 2.5 times the footprint of the resonators 
with a magneto-electric tensor element which amounts to $63 \%$ of the electric dipole one. ${ }^{19}$ This hints towards the possibilityto design small pixels where the circular dichroism would scale with the number of U-shaped resonators in the pixel. In this work we explain how plasmon resonances with magneto-electric coupling can be used to encode images in circular polarization with contrast reversal depending on the direction of observation and we illustrate our study with the realization of an image of Leonardo Da Vinci's masterpiece, La Joconde, displayed at the Louvres Museum in Paris.

The polarization state of the light scattered by an isolated pseudo-chiral resonator excited at a mode presenting magneto-electric coupling can be obtained from the knowledge of its polarizability tensor. The polarizability tensor relates the electric and magnetic moments, $\vec{p}$ and $\vec{m}$ to the excitation electric and magnetic fields $\vec{E}^{e x c}$ and $\vec{H}^{e x c} \cdot{ }^{18,24-26}$ The magnetic dipole moments are associated with plasmon modes for which the current distributions exhibit current loops (see Figure 1(a)). Without loss of generality, we neglect in this study the electric quadrupole moments ${ }^{19,21,29,30}$ and assume that the dependence on the gradients of the fields can be described by an effective polarizability tensor $\widetilde{\alpha}:{ }^{19,30}$

$$
\left[\begin{array}{c}
\vec{p} \\
\vec{m}
\end{array}\right]=\left[\begin{array}{cc}
\widetilde{\alpha}^{e e} & \widetilde{\alpha}^{e m} \\
-\left(\widetilde{\alpha}^{e m}\right)^{T} & \widetilde{\alpha}^{m m}
\end{array}\right]\left[\begin{array}{c}
\vec{E}^{e x c} \\
\vec{H}^{e x c}
\end{array}\right]
$$

where $\widetilde{\alpha}^{e e}, \widetilde{\alpha}^{m m}$ and $\widetilde{\alpha}^{e m}$ are the electric, magnetic and magneto-electric polarizability tensors corrected for the gradients of the fields, respectively. Considering the orientation of the dipole moments in a U-shaped resonator (Figure 1(a)) and assuming point-like dipole moments, it is possible to determine the total electric field scattered in the plane containing the bottom arm of the resonators (plane $(y, z))$ for incident light propagating in the plane $(y, z)$ at an angle $\theta$ from the axis $z$ ( Figure $1(\mathrm{~b})$ ). When the incident light is p-polarized (i.e. in the $(y, z)$ plane) the electric field scattered in the same direction as the incident one can be decomposed in p and s contributions (see Supporting Information S.I.1) : 


$$
\left[\begin{array}{c}
E_{p p}^{s c a t t} \\
E_{s p}^{s c a t t}
\end{array}\right]=\frac{k^{2}}{4 \pi \epsilon_{o}}\left[\begin{array}{c}
\alpha_{y y}^{e e} \cos ^{2}(\theta) \\
-\frac{i}{2 c} \alpha_{y z}^{e m} \sin (2 \theta)
\end{array}\right]
$$

and when the incident light is s-polarized (i.e. along $x$ ) one obtains :

$$
\left[\begin{array}{c}
E_{p s}^{s c a t t} \\
E_{s s}^{s c a t t}
\end{array}\right]=\frac{k^{2}}{4 \pi \epsilon_{o}}\left[\begin{array}{c}
\frac{i}{2 c} \alpha_{y z}^{e m} \sin (2 \theta) \\
\alpha_{x x}^{e e}+\alpha_{z z}^{m m} \sin ^{2}(\theta)
\end{array}\right]
$$

where $k$ is the wavenumber, $c$ the celerity of light, $\epsilon_{o}$ the dielectric permittivity of vacuum. The third component on the vector being along the propagation direction in this basis is equal to zero and was omitted in Equations (2) and (3). The $\alpha_{i j}$ are the $(i, j)=(x, y, z)$ elements of the different polarizability tensors. The total transmitted field should also contain the contribution of the incident field. However, in the following we will focus on the conversion of polarization and on observation between crossed-polarizers for which the incident field is filtered out. As a consequence of the presence of the magneto-electric coupling element $\alpha_{y z}^{e m}$ in the polarizability tensor yielding the $E_{p s}^{s c a t t}$ and $E_{s p}^{s c a t t}$ components, the transmitted light is elliptically polarized, except at normal incidence, and the handedness of the polarization rotation changes of sign when the angle of incidence is changed from $\theta$ to $-\theta$ as a consequence of the $\sin (2 \theta)$ factor. This property is maintained in arrays of resonators (see schematic of Figure 1(b)). ${ }^{28,31}$ It can also be seen from Equations (2) and (3) that whatever the incident linear polarization used, p or s, the radiated light transmitted in a linear polarization orthogonal to the incident one is proportional to $\sin ^{2}(2 \theta)\left|\alpha_{y z}^{e m}\right|^{2}$ (Figure 1(c)). For light propagating in the plane containing the lateral arms of the resonators, no light is expected to be observed between crossed linear polarizers.

The plasmonic resonators investigated here are sketched in Figure 1(a). They were fab- 


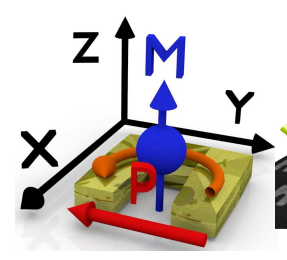

(a)

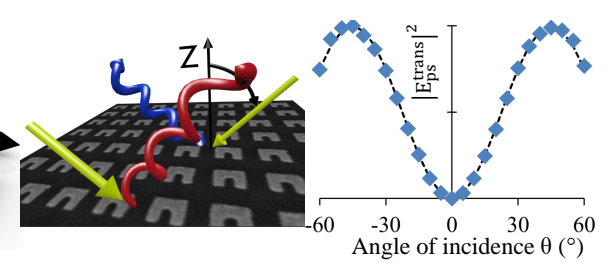

(b)

(c)

Figure 1: (a) Sketch of the dipole moments excited in an isolated U-shaped resonator associated with the current distribution at resonance. (b) Influence of an array of $U$-shaped resonators on the transmitted polarization. (c) Normalized variation of intensity between crossed linear polarizers $\left|E_{p s}^{s c a t t}\right|^{2}$ with respect to the angle of incidence $\theta$. The symbols present the values calculated at a wavelength of $770 \mathrm{~nm}$ using Finite Element Methods (FEM). They follow the $\sin ^{2}(2 \theta)$ variation (line) expected in presence of magneto-electric coupling. See S.I.2 for a description of the FEM calculations.

ricated with electron beam lithography followed by a conventional lift-off method. A 130 nm thick layer of PMMA was spin-coated on a clean glass substrate (BK7) and a thin layer of aluminum was thermally evaporated on the PMMA to avoid any charging effect during the exposure with electrons. After exposure and before the development of the resist, this conductive layer was removed in a $\mathrm{KOH}$ bath. After development, a $4 \mathrm{~nm}$ chromium layer was evaporated to ensure the adhesion of the $40 \mathrm{~nm}$ gold layer on the glass substrate. The resist was then lifted-off with acetone, and the substrate subsequently rinsed in ethanol to reveal the $U$-shaped scatterers. Such resonators exhibit a resonance with magneto-electric coupling near $770 \mathrm{~nm}$ excited by the component of the electric field of light parallel to the $y$ axis. To investigate the dependence of the scattered intensity in circular polarization as a function of the resonators density, we have realized a palette where pixels consisted in a $3 \times 3$ matrix of resonators with a total footprint of $1.4 \times 1.4 \mu m^{2}$ per pixel (Figure $2(\mathrm{a})$ ). The number of U-shaped resonators in each pixel varied between 1 and 9. When only U-shaped resonators are used, the transmittance of the surface scales inversely with the number of resonators. To keep the transmittance constant across all pixel configurations, the missing resonators were replaced by circular resonators in each pixel and all the surfaces in between the palette were also filled with circular resonators (Figure 2(a)). Circular resonators do not show any optical activity (i.e. $\alpha^{e m}=0$ ), but they scatter unpolarized light in an amount 
comparable to that of U-shaped resonators in direct transmission conditions (see also S.I.2). The purpose of adding the circular resonators is to strengthen the camouflage of the structure at normal incidence. In this way an encoded pattern observed with unpolarized light will be invisible whereas it will be clearly visible when seen between crossed polarizers. Figure 2(b) shows that the absorbance was approximately constant across all configurations and that the U-shaped resonators could not be distinguished from the circular ones with a diffraction limited resolution. In contrast, Figure 2(c) presents a microscopy image of the palette of Figure 2(b) taken between crossed linear polarizers at an incidence $\theta \approx 30^{\circ}$ around the axis $x$ (see Figure $1(\mathrm{~b})$ ). It can be seen that light was transmitted in these conditions in the area containing the U-shaped resonators, with a luminance scaling approximately with the number of U-shaped resonators in each pixel. The topmost row of the palette in Figure 2(c) contains arrays of pixels containing $3 \times 3$ resonators among which 3 U-shaped resonators but with different positions in each pixel. The intensity transmitted at oblique incidence between crossed linear polarizers shows some variations with the organization of the U-shaped resonators inside a pixel. This suggests that the luminance can be further engineered by organizing the resonators within each pixel. The influence caused by the distribution of the U-shaped resonators within the pixel is of second order compared with the influence of the actual number of $\mathrm{U}$ shaped resonators within the pixel. Therefore, in the context of this work, attention will be paid on the effects caused by the number of U-shaped resonators per pixel.

To investigate more quantitatively the influence of the number of U-shaped resonators per pixel on the circular dichroism observed at oblique incidence, accurate measurements of the circular dichroism have been performed on the palette. We define here the circular dichroism, $C D$ as the transmittance difference between right, $T_{R C P}$, and left circular polarization $T_{L C P}$ for unpolarized incident light. The symmetry upon time reversal states that this definition yields the same result as measuring the difference of the total transmitted intensities for incident right and left circular polarizations (see S.I.3). Figure 3 presents the images in 

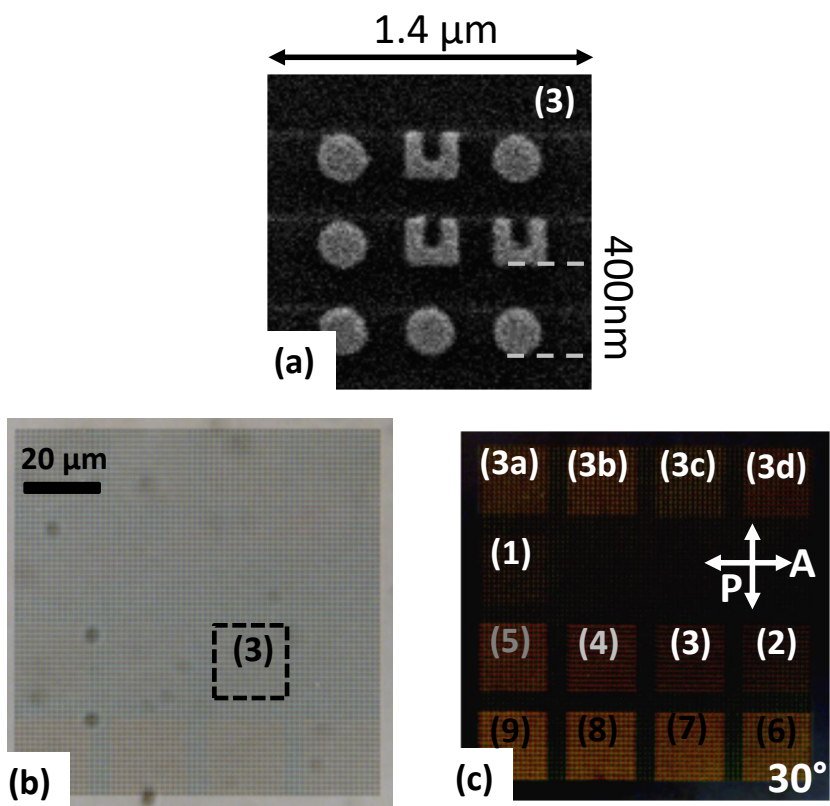

Figure 2: (a) SEM image of a pixel with $3 \mathrm{U}$-shaped resonators and 6 disks placed on a square lattice with a constant of $400 \mathrm{~nm}$. (b) Optical microscopy image in transmission at normal incidence $(\times 50)$ using unpolarized white light of the palette made of U-shaped resonators and disks. The area containing pixels with 3 U-shaped resonators has been highlighted with dotted lines for illustration purposes. (c) Same palette as in (b) but observed between crossed polarizers at oblique incidence. The orientations of the linear polarizer $(\mathrm{P})$ and linear analyzer (A) are indicated in the figure. The number of U-shaped resonators per pixels is indicated for each array (labeled 1 to 9). On the top row, 4 different configurations of U-shaped resonators are displayed (labeled 3a to $3 \mathrm{~d}$ ). 
circular dichroism measured in transmission (objective $\times 50$ ) at a wavelength of $650 \mathrm{~nm}$ on the palette of Figure 2(b). The Multimodal imaging Mueller polarimetric microscope used to measure the circular dichroism is described in details in S.I.3 and is based on the system described in Ref. 32. Two different directions of propagation in the plane $(y, z)$ were used corresponding to $\theta=-35^{\circ}$ and $\theta=+35$. The $C D$ can also be calculated from Equations 2 and 3 by projection of the transmitted fields onto the basis of circular polarizations. For unpolarized incident excitation, the total intensity in each circular polarization is then obtained by adding the intensities obtained for $\mathrm{p}$ - and s-polarized incident light, yielding (see S.I.1) :

$\mathrm{CD}=\mathrm{T}_{R C P}-T_{L C P}=-\frac{1}{c}\left(\frac{k^{2}}{4 \pi \epsilon_{o} c}\right)^{2} R e\left\{\left(\alpha_{y y}^{e e} \cos ^{2}(\theta)+\alpha_{x x}^{e e}+\alpha_{z z}^{m m} \sin ^{2}(\theta)\right) \alpha_{y z}^{e m *}\right\} \sin (2 \theta)(4)$

No noticeable circular dichroism should be measured neither at normal incidence nor at oblique incidence with light propagating in a plane containing the lateral arms of the resonators $((x, z)$ plane). In contrast, when light propagates at oblique incidence and in the plane containing the bottom arm of the resonators $((y, z)$ plane), a significant signal should be observed in circular dichroism scaling as the magneto-electric coupling element $\alpha_{y z}^{e m}$. In addition, sign reversal of the $C D$ with respect to the direction of propagation with respect to the normal to the surface, i.e. to the sign of $\theta$, is expected because of the factor $\sin (2 \theta)$ present in Equation (4). This sign reversal was indeed observed in the experimental measurements performed at a wavelength of $650 \mathrm{~nm}$ and presented in Figure 3. The values of $C D$ were measured for each type of pixel and the two angles of incidence $+35^{\circ}$ and $-35^{\circ}$. They are plotted in Figure 3 as a function of the number of U-shaped resonators in each pixel. The circular dichroism increased steadily with the number of U-shaped resonators and the values were exactly opposite when changing the angle of incidence $\theta$ from $+35^{\circ}$ to $-35^{\circ}$. To confirm these observations, the circular dichroism of arrays of $3 \times 3$ resonators 
was calculated with an electromagnetic simulator based on finite element methods (HFFS by ANSYS, see S.I.2). An excellent agreement between measured and calculated values can be observed in Figure 3. This shows that it is possible to encode images in circular polarization using pseudo-chiral U-shaped resonators.

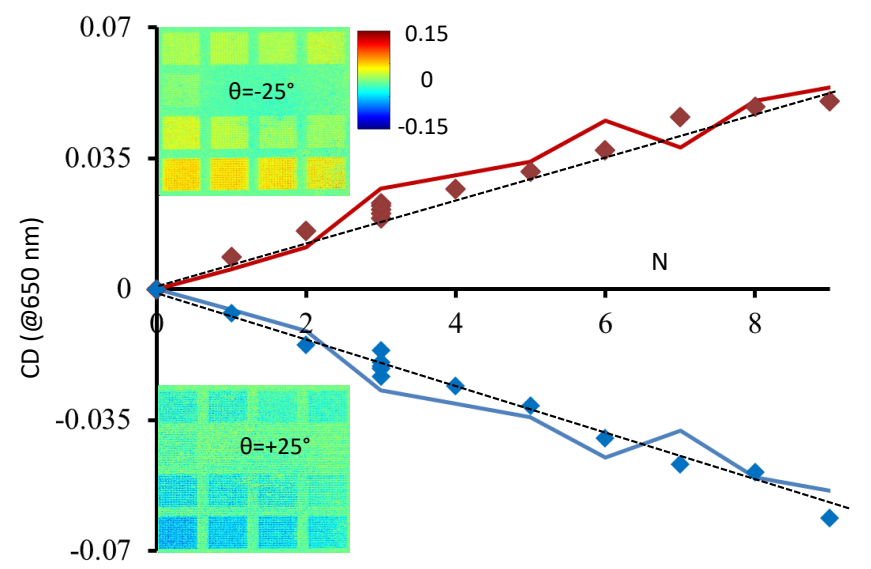

Figure 3: Circular dichroism measured on the palette (symbols) and calculated by Finite Element Methods (lines) for different numbers $\mathrm{N}$ of pseudo-chiral resonators per pixel at $+35^{\circ}$ (blue line and symbols) and $-35^{\circ}$ (red line and symbols) of incidence. The dotted lines are simple linear interpolations of the measured values. The inset shows the images of the palette (x50) observed in circular dichroism (encoded in false colors).

It can be inferred from Equations (2) and (3), on the one hand, and Equation (4), on the other hand, that both color and luminance perceived between crossed linear polarizers scale directly as the absolute value of the circular dichroism since both linear and circular dichroism scale as $\alpha_{y z}^{e m} \sin (2 \theta)$. The interest of using crossed linear polarizers is that it is easy to find efficient broad spectral band linear polarizers. The encoded image would appear only at oblique incidence as a bright image on a dark background created by the crossed polarizers provided that the absorbance of the U-shaped resonators is compensated for by achiral plasmonic disks (Figure 2(a)). The perceived color and luminance were calculated from the calculated spectral response of the circular dichroism for each type of pixel configuration and different angles of incidence. Figure 4 shows the spectral dependence of the calculated circular dichroism for a pixel containing 9 U-shaped resonators. At normal incidence no circular dichroism is obtained whereas as soon as the angle of incidence is tilted from the 
normal incidence, circular dichroism appears and increases with the angle of incidence. It can be noted that the maximum of $\mathrm{CD}$ is observed near $770 \mathrm{~nm}$ which is the position of the resonance exhibiting magneto-electric coupling. The corresponding colors were calculated from the spectral response of the pixel for each angle of incidence by using the conventional color matching functions $(\mathrm{CMF})$ and using the color response of the tungsten-halogen lamp used in our microscope. The colors in circular dichroism associated with each angle of incidence are plotted on top of the graph in Figure 4. A very good agreement between calculated (Figure 4) and observed values (Figure 2(c)) was obtained for an incidence of $\theta=35^{\circ}$. A brownish color was calculated and observed as seen in Figure 2(c) and (d) because of the combination of the resonance in the near infrared and of the interband transitions of gold in the blue-green part of the visible spectrum of light. It was also observed (not shown here) that the luminance scaled with both the number of resonators and the angle of incidence. These variations were in the same proportion for each pixel which ensures that the effect is robust and that the image contrast encoded in circular polarization would be maintained for any angle of observation (with the exception of normal incidence). These results are completely consistent with the presence of magneto-electric coupling in mostly independent U-shaped resonators with an extinction cross-section of the order of magnitude of 2.5 times the surface of each resonator placed on a lattice of 2.8 times the surface of each resonator.

To illustrate the potential of pseudo-chiral resonators to encode image contrasts with a resolution of $1.4 \mu \mathrm{m}$ in circular polarization, we have transformed a grayscale image of a detail of the painting La Joconde in a luminance image observed in circular polarization dichroism. The grayscale image of Figure 5(a) was first pixelized with ten values of gray to obtain Figure 5(b). Each pixel was then replaced by an array of $3 \times 3$ resonators with $\mathrm{U}$ - or circular shape. The number of U-shaped resonators per array ranges between 0 and 9 which defines the 10 values of luminance used to pixelize the painting (see Figure 2(c)). After e-beam lithography, the metasurface containing the covert image of La Joconde was 


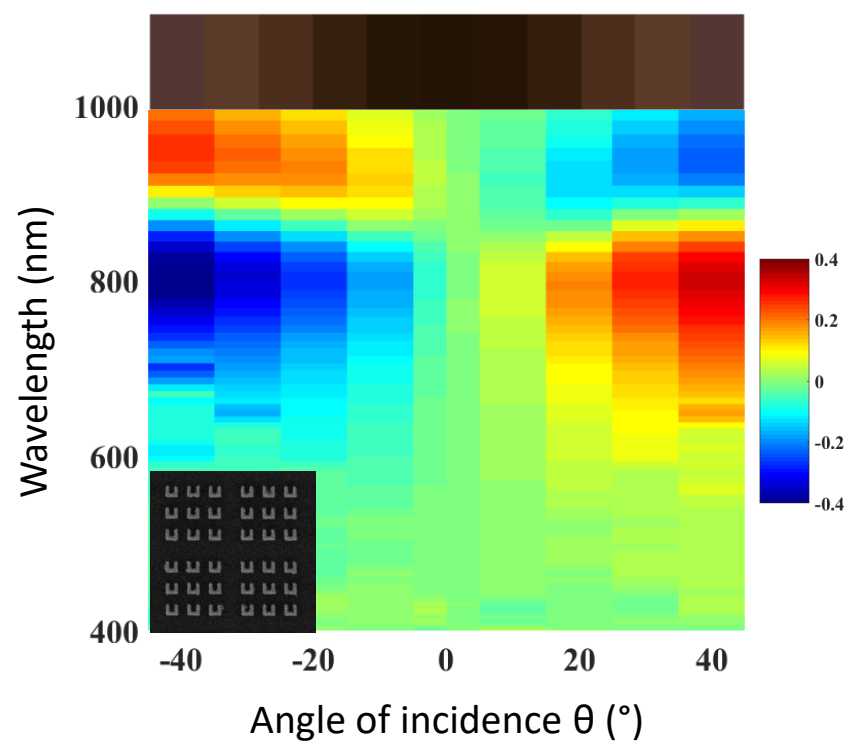

Figure 4: (a) Spectral dependence of the circular dichroism as a function of the angle of incidence for pixels containing 9 U-shaped resonators. The value of the circular dichroism increases with incidence. The color perceived in circular dichroism is displayed on top of the graph.

observed in unpolarized optical microscopy (Figure 5(c)). The grayscale image of Figure 5(b) was concealed and only a darker area could be observed at the location of the pixels. Without adding circular resonators, the image would have been visible under unpolarized light illumination (see S.I.4). The inset in Figure 5(c) shows a magnified image (using SEM) of 2 pixels with different compositions. The metasurface was then observed between linear crossed polarizers. The colors of the image were corrected by a white balance performed on the substrate imaged between parallel linear polarizers and a dark correction performed on the substrate imaged between crossed linear polarizers. When observed at normal incidence no image could be seen (Figure 5(d)). As soon as the metasurface was tilted about $x$ axis, the bright image of La Joconde was revealed on a dark background (Figure 5(e)). Indeed, at oblique incidence only the U-shaped resonators induced circular polarization that was revealed between the cross-polarizers while the disks remained dark. The colors were also in good agreement with those calculated in Figure 4(b).

To confirm that the image can truly be encoded in circular polarization, a second metasurface was realized where the pixels were obtained by replacing the disks by upside-down 

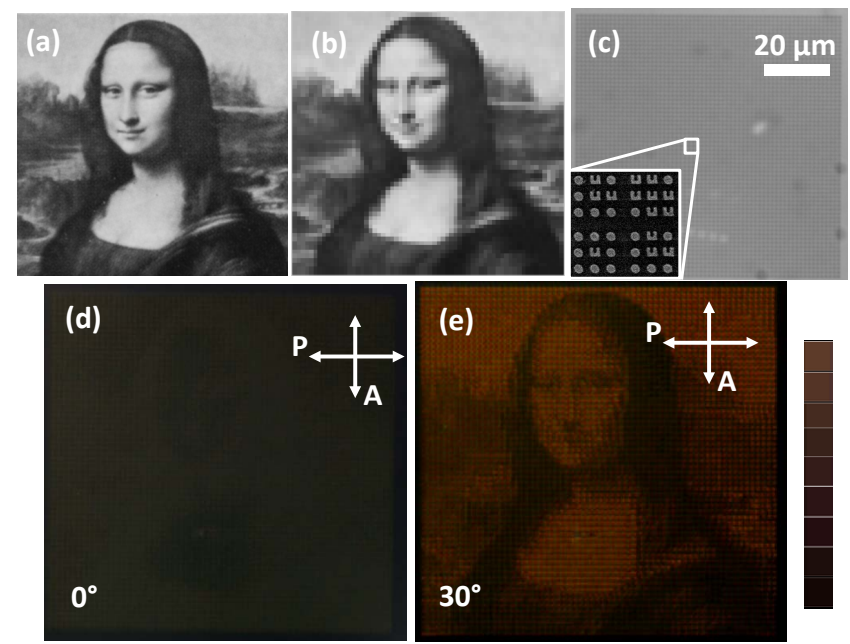

Figure 5: Illustration of the transformation of a (a) grayscale image of a detail of the painting La Joconde to $(\mathrm{b})$ the same image after pixelization $(50 \times 70$ pixels $)$ and reduced to 10 grayscale levels. (c) Metasurface observed in optical microscopy where each gray level has been replaced by a $1.4 \times 1.4 \mu \mathrm{m}^{2}$ pixels containing array of 9 scatterers $(U$-shaped and circular, see inset). (d) Image observed between crossed linear polarizer (P) and analyzer (A) at an incidence $\theta=0^{\circ}$ and (e) at an incidence $\theta \approx 30^{\circ}$. The colorbar is extracted from Figure 4.

U-shaped resonators as illustrated in Figure 6 (a). It can be seen that in unpolarized transmission the image was concealed (Figure 6 (a)). But now the image was also barely distinguishable between crossed linear polarizers at oblique incidence as well, as shown in Figure 6 (b) in contrast with what was observed when the pixels were coded using U-shaped resonators and disks (compare with Figure 5(e)). However, at oblique incidence the covert image of La Joconde was revealed in circular dichroism. Figure 6(c) presents the image observed in circular dichroism at $650 \mathrm{~nm}$. Because of the combination of U-shaped resonators and upside-down U-shaped resonators, the image appeared in one particular circular dichroism on a background with opposite circular dichroism and the contrast in circular dichroism was reversed upon change of the angle of incidence from $+35^{\circ}$ to $-35^{\circ}$ as displayed in Figure 6(c)). Although the observations were made in transmission in the present work, similar effects would be observed in reflection mode. Indeed, circular dichroism and its reversal with propagation direction has been described on arrays of U-shaped resonators. ${ }^{18,28}$

These results evidence that pseudo-chiral resonators present an additional degree of security compared to images encoded in linear polarizations which would not possibly show such 


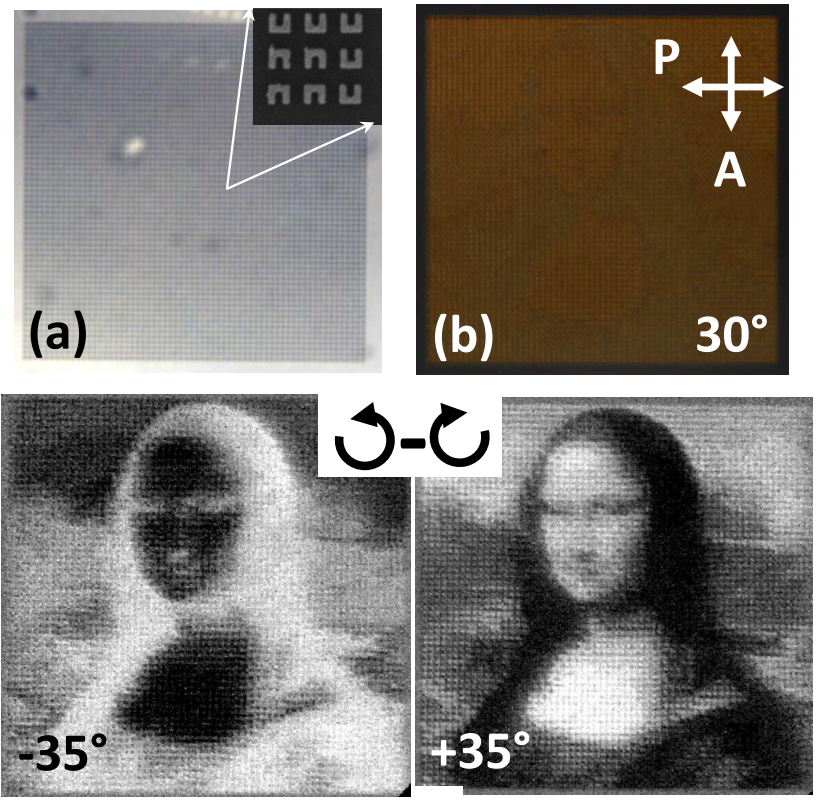

(c)

Figure 6: (a) Unpolarized transmittance image of La Joconde with pixels encoded with right and upside-down U-shaped scatterers (see inset). (b) Same metasurface observed between crossed linear polarizers at oblique incidence. (c) Circular dichroism measured in transmission at a wavelength of $650 \mathrm{~nm}$ as a function of the angle of incidence. The symmetric grayscale ranges from negative (black) to positive (white) values of circular dichroism. 
contrast reversal upon angle of observation changes. Different colors may be obtained by using different materials expanding the accessible resonances to the blue part of the spectrum and combining different types of resonators in the same pixel. Better contrast variations may also be obtained by increasing the number of resonators in each pixel, to the detriment of lateral resolution.

\section{CONCLUSION}

We have shown how contrasts of images could be encoded in circular dichroism by using the magneto-electric coupling yielded by plasmonic resonances of pseudo-chiral metasurfaces. By modeling the optical response of pseudo-chiral plasmonic resonators using point-like electric and magnetic dipoles, we have derived the equations yielding the dependency of the intensity observed in circular dichroism and between crossed polarizers of an image built from pixels containing U-shaped resonators. We have confirmed experimentally the theoretical results using a palette of 10 levels of contrasts. The possibility to design covert images revealed only between crossed polarizers and with contrast reversal in circular dichroism was shown by creating a pseudo-chiral metasurface containing a representation of La Joconde.

\section{Acknowledgement}

J.B. acknowledges funding from a Marie Sklodowska-Curie Fellowship.

\section{Author Contributions}

T.S.H. Yoo and E. Garcia-Caurel developped and did the measurements using the multimodal imaging microscope. J. Berthelot particpated in the measurements using linear polarization. G. Guida did the numerical simulations. D. Demaille did all the processes involved in the e-beam lithography for the sample preparation. N. Bonod and B. Gallas developped the 
models and did the article preparation.

\section{Supporting Information}

Supporting Information contains :

S.I.1 : description of the multipole model and calculations yielding to the equations presented in this article

S.I.2 : description of the numerical simulations and optical properties of the disk-shaped resonators

S.I.3 : description of the multimodal imaging microscope definition of circular dichroism S.I.4 : descritpion of the realization of an image with only U-shaped resonators

\section{References}

(1) Enoch, S.; Bonod, N. Plasmonics : From Basics to Advanced Topics, Springer, Berlin, 2012

(2) Proust, J.; J.; Bedu, F.; Gallas, B.; Ozerov, I.; Bonod, N. All-Dielectric Colored Metasurfaces with Silicon Mie Resonators. ACS Nano 2016, 10, 7761-7767

(3) Flauraud, V.; Reyes, M.; Paniagua-Domínguez, R.; Kuznetsov, A.I.; Brugger, J. Silicon Nanostructures for Bright Field Full Color Prints. ACS Photonics 2017, 4, 1913-1919

(4) Sun, S.; Zhou, Z.; Zhang, C.; Gao, Y.; Duan, Z.; Xiao, S.; Song, Q. All-Dielectric Full-Color Printing with TiO2 Metasurfaces. ACS Nano 2017, 11, 4445-4452

(5) Tan, S.J.; Zhang, L.; Zhu, D.; Goh, X.M.; Wang, Y.M.; Kumar, K.; Qiu, C.-W.; Yang, J.K.W. Plasmonic Color Palettes for Photorealistic Printing with Aluminum Nanostructures. Nano Lett. 2014, 14, 4023-4029 
(6) Kumar, K.; Duan, H.; Hegde, R.S.; Koh, S.C.W.; Wei, J.N.; Yang J.K.W. Printing colour at the optical diffraction limit. Nat. Nanotechnol. 2012, 7, 557-561

(7) Finlayson, E.D.; Hooper, I.R.; Lawrence, C.R.; Heath, M.; Anderson, D.; Sambles, J.R.; Vukusic P. Covert Images Using Surface Plasmon-Mediated Optical Polarization Conversion. Adv. Optical Mater. 2018, 20, 1700843

(8) Duempelmann, L.; Luu-Dinh, A.; Gallinte, B.; Novotny, L. Four-fold Color Filter Based on Plasmonic Phase Retarder. ACS Photon. 2015, 3, 190-195

(9) Zijlstra, P.; Chon, J. W. M.; Gu, M. Five-dimensional optical recording mediated by surface plasmons in gold nanorods. Nature 2009, 459, 410-413

(10) Mathilde Makhsiyan, Patrick Bouchon, Julien Jaeck, Jean-Luc Pelouard, Riad Haida, Shaping the spatial and spectral emissivity at the diffraction limit. Appl. Phys. Lett. 2015, 10\%, 251103

(11) Kristensen,A.; Yang, J.K.W.; Bozhevolnyi, S.I.; Link, S; Nordlander, P.; Halas, N.J.; Mortensen, N.A. Plasmonic colour generation. Nat. Rev. Mater. 2016, , 16088

(12) Wegener, M.; Zheludev, N. I. Artificial Chiral Materials. Journal of Optics A: Pure and Applied Optics 2009, 11, 070201

(13) Fan, Z.; Govorov, A. O. Plasmonic Circular Dichroism of Chiral Metal Nanoparticle Assemblies. Nano lett. 2010, 10, 2580-2587

(14) Kuzyk, A.; Schreiber, R.; Fan, Z.; Pardatscher, G.; Roller, E.-M.; Hogele, A.; Simmel, F. C.; Govorov, A. O.; Liedl, T. DNA-based Self-Assembly of Chiral Plasmonic Nanostructures With Tailored Optical Response. Nature 2012, 483, 311-314

(15) Collins, J.T.; Kuppe, C.; Hooper, D.C.; Sibilia, C.; Centini, M.; Valev, V.K. Chirality and Chiroptical Effects in Metal Nanostructures : Fundamentals and Current Trends. Adv. Optical Mater. 2017, 1700182 
(16) Valev, V. K.; Baumberg, J.J.; De Clercq, B.; Braz, N.; Zheng, X.; Osley, E.J.; Vandendriessche, S.; Hojeij, M.; Blejean, C.; Mertens, J.; Biris, C.G.; Volskiy, V.;Ameloot, M.; Ekinci, Y.; Vandenbosch, G.A.E.; Warburton, P.A.; Moshchalkov, V.V.; Panoiu, N.C.; Verbiest, T. Nonlinear Superchiral Meta-Surfaces: Tuning Chirality and Disentangling Non-Reciprocity at the Nanoscale. Adv. Mater. 2014, 26, 4074âĂŞ4081

(17) Kolkowski, R.; Petti, L.; Rippa, M.; Lafargue, C.; Zyss, J. Octupolar Plasmonic MetaMolecules for Nonlinear Chiral Watermarking at Subwavelength Scale. ACS Photonics 2015, 2, 899âĂ\$̧906

(18) Sersic, I.; Tuambilangana, C.; Kampfrath, T.; Koenderink, A.F. Magnetoelectric Point Scattering Theory for Metamaterial Scatterers. Phys. Rev. B 2011, 83, 245102

(19) Proust, J.; Bonod, N.; Grand, J.; Gallas, B. Optical Monitoring of the Magnetoelectric Coupling in Individual Plasmonic Scatterers. ACS Phot. 2016, 3, 1581-1588

(20) Alizadeh, M.H.; Reinhard, B.M. Plasmonically Enhanced Chiral Optical Fields and Forces in Achiral Split Ring Resonators. ACS Phot. 2015, 2, 361-368

(21) Arango, F. B.; Coenen, T.; Koenderink,A. F. Underpinning Hybridization Intuition for Complex Nanoantennas by Magnetoelectric Quadrupolar Polarizability Retrieval. ACS Phot. 2014, 1, 444-453

(22) Saadoun, M.M.I.; Engheta, N. A Reciprocal Phase Shifter Using Novel Pseudochiral or $\Omega$ Medium. Microwave Opt. Technol. Lett. 1992, 5, 184-188

(23) Plum, E.;Liu, X.-X.; Fedotov, V.A.; Chen, Y.; Tsai, D.P.; Zheludev, N.I. Metamaterials: Optical Activity without Chirality. Phys. Rev. Lett. 2009, 102, 113902

(24) Hentschel, M.; Schaferling, M.; Weiss, T.; Liu, N.; Giessen, H. Three-Dimensional Chiral Plasmonic Oligomers. Nano lett. 2012, 12, 2542-2547 
(25) I. V. Lindell, A. Sihvola, and S. Tretyakov, Electromagnetic Waves in Chiral and BiIsotropic Media (Artech House, 1994)

(26) R. E. Raab and O. L. de Lange, Multipole Theory in Electromagnetism: Classical, Quantum, and Symmetry Aspects, with Applications (Oxford University , 2005)

(27) Sersic, I.; Frimmer, M.; Verhagen, E.; Koenderink, A.F. Electric and Magnetic Dipole Coupling in Near-Infrared Split-Ring Metamaterial Arrays. Phys. Rev. Lett. 2009, 103, 213902

(28) Guth, N.; Gallas, B.; Rivory, J.; Grand, J.; Ourir, A.; Guida, G.; Abdeddaim, R.; Jouvaud, C.; de Rosny, J. Optical Properties of Metamaterials: Influence of Electric Multipoles, Magnetoelectric Coupling, and Spatial Dispersion. Phys. Rev. B 2012, 85, 115138

(29) Cho, D.J.; Wang, F.; Zhang, X.; Shen, Y.R. Contribution of The Electric Quadrupole Resonance In Optical Metamaterials. Phys. Rev. B 2008, 78, 121101(R)

(30) Varault, S.; Rolly, B.; Boudarham, G.; Demesy, G.; Stout, B.; Bonod, N. Multipolar Effects On The Dipolar Polarizability Of Magneto-Electric Antennas. Opt. Express 2013, 21, 16444-16454

(31) Sersic, I.; Frimmer, M.; Verhagen, E.; Koenderink, A.F. Electric and Magnetic Dipole Coupling in Near-Infrared Split-Ring Metamaterial Arrays. Phys. Rev. Lett. 2009, 103, 213902

(32) Yoo, S. H.; Ossikovski, R. and Garcia-Caurel, E. Experimental Study of Thickness Dependence of Polarization and Depolarization Properties of Anisotropic Turbid Media Using Mueller Matrix Polarimetry and Differential Decomposition. Appl. Surf. Sci. 2017, 421, 870-877. 


\section{For Table of Contents Use Only}

Circularly polarized images with contrast reversal using pseudo-chiral metasurfaces

Thomas Sang Hyuk Yoo, Johann Berthelot, Geraldine Guida, Dominique Demaille, Enric Garcia-Caure, Nicolas Bonod, Bruno Gallas

This graphic illustrates a U-shaped resonators with the moments at the origin of the observed circular dichroism. It presents an image of a surface with 4 pixels containing different numbers of resonators to illustrate the spatial and strength control of the generation of circular dichroim. Then there are two images of MonaLisa presented as filtered by circular polarizers emerging from the surface.

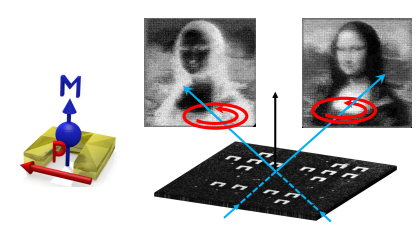

